\title{
1 Basin-scale transmissivity and storativity estimation using hydraulic tomography
}

2 Kristopher L. Kuhlman, Andrew C. Hinnell, Phoolendra K. Mishra,

4 University of Arizona, Department of Hydrology \& Water Resources, 1133 East James E. Rodgers Way, Tucson, AZ 85721.

\section{Abstract:}

7 While tomographic inversion has been successfully applied to laboratory- and field-scale

8 tests, here we address the new issue of scale that arises when extending the method to a

9 basin. Specifically, we apply the hydraulic tomography concept to jointly interpret four

10 multi-well aquifer tests in a synthetic basin to illustrate the superiority of this approach to a

11 more traditional Theis analysis of the same tests. Transmissivity and storativity are

12 estimated for each element of a regional numerical model using the geostatistically-based

13 SSLE inverse solution method. We find that hydraulic tomography inversion is an effective

14 strategy for incorporating data from potentially disparate aquifer tests into a basin-wide

15 aquifer property estimate. The robustness of the SSLE algorithm is investigated by

16 considering the effects of noisy observations, changing the variance of the true aquifer

17 parameters, and supplying incorrect initial and boundary conditions to the inverse model.

18 Ground water flow velocities and total confined storage are used as metrics to compare true

19 and estimated parameter fields; they quantify the effectiveness of hydraulic tomography

20 and SSLE compared to a Theis solution methodology. We discuss alternative software that

21 can be used for implementing tomography inversion. 
24 Managing ground water resources requires knowledge of aquifer property distributions, 25 since they affect water movement and solute transport. This understanding is often 26 developed and tested with regional numerical ground water flow models, which are used 27 for simulation, prediction, and scenario analysis. Regional models facilitate long-term 28 management of water resources, where they can be used for both evaluation and mitigation 29 of supply and quality issues.

31 In ground water model calibration we seek to best represent a complex natural system with 32 an idealized numerical model at the appropriate scale of interest. The scale depends on the 33 intended use of the calibrated model (e.g., flow vs. transport predictions) and the desired 34 detail needed in the predictions. Many regional ground water studies do not attempt to 35 build detailed heterogeneity into large scale (tens to hundreds of kilometers)flow models, 36 due to the prohibitive costs of detailed sampling over large areas and the computational

37 limits on calibrating multi-scale heterogeneity in the model. Regional geologic or 38 hydrologic units are often treated as zones, assumed to be homogeneous with a single 39 effective parameter value (e.g., Barlebo et al. [2004]). This zoned representation may offer 40 computational advantages, but it can only yield large-scale effective properties, which are 41 best for predicting "ensemble" behaviors of a ground water system [Yeh, 1992; Yeh et al., 42 2007].

44 In regional studies that include local-scale heterogeneity (i.e., heterogeneity smaller than 45 the hydrologic unit, at the scale of several model cells), the parameter distribution is often 
67 To improve our ability to adequately characterize and solve inverse ground water 68 problems, we propose utilizing hydraulic tomography (HT). Many researchers have shown

estimated from a steady-state or pre-development head distribution (e.g., Yeh and Mock [1996]). Heterogeneous transmissivity fields are estimated by manually adjusting parameter values in model cells or zones to match simulated and observed hydraulic heads. More advanced approaches use automated calibration algorithms (e.g., PEST [Doherty, 2007] or UCODE [Poeter et al., 2005]) to minimize the residual between observed and simulated heads [Barlebo et al., 2004]. Steady-state calibrations are limited to estimating transmissivity $(T)$, and few regional studies attempt to calibrate ground water flow models using transient head measurements due to the large increase in complexity and computational effort.

Basin-scale transient model calibrations are often ill-posed and non-unique due to difficulties collecting the necessary and sufficient information to make an inverse problem well-posed [Yeh et al., 2007]. As a result, there are many non-unique parameter distributions that equally fit sparse head observations. In other words, traditional inverse modeling efforts often yield ambiguous aquifer characterization. Because of the uncertainty inherent in aquifer parameter and boundary condition characterization, many modelers have developed misleading predictive models of ground water flow and contaminant migration. Because of this, some have seriously questioned the ability to validate ground water flow models at all [Konikow and Bredehoeft, 1992; Oreskes et al., 1994; Bredehoeft, 2003]. 
69 it can be used to characterize heterogeneous hydraulic properties, including Tosaka et al.

70 [1993], Gottlieb and Dietrich [1995], Vasco et al. [2000], Yeh and Liu [2000], Bohling et

71 al. [2002], Brauchler et al. [2003], and Zhu and Yeh [2005 and 2006]. HT involves

72 collecting responses throughout an aquifer due to a sequence of overlapping aquifer tests,

73 then calibrating a heterogeneous ground water flow model using the observed responses

74 from all the tests. Multiple sets of aquifer tests and their observed responses improve the

75 inverse problem, since tests cross-validate each other. As a result, the estimated hydraulic

76 property fields become more detailed and less uncertain than those computed from a single

77 set of data.

79 HT has been applied successively to small-scale synthetic aquifers [Yeh and Liu, 2000; Zhu 80 and Yeh, 2005 and 2006; Hao et al., 2008], laboratory sandboxes [Liu et al., 2002; Liu et 81 al., 2007; Illman et al., 2007], and plot-scale fields [Vesselinov et al., 2001; Bohling et al., 82 2007; Straface et al., 2007; Li et al, 2007]. In these small-scale studies it is possible to 83 stress the entire domain with each pumping well, providing new information throughout 84 the domain from each pumping event. We propose using regional-scale HT to estimate $T$ 85 and storativity $(S)$ distributions for a regional flow model, where the main new challenge is 86 determining how to adequately stress the entire aquifer. Unlike smaller-scale applications 87 of HT, it is not possible to pump a single well causing a response throughout the aquifer; 88 both the pumping rate and test length would be unreasonably large. Realistically, a single 89 aquifer test can only stress a portion of a large aquifer and only cause measurable 90 drawdown in a subset of a basin-wide observation network. At the regional scale, we 91 reformulate $\mathrm{HT}$ as an interference problem; the head distribution due to multiple 
92 simultaneous pumping wells is observed using a monitoring well network as might be

93 found in a municipal water supply or remedial well field (off-duty pumping wells can serve

94 as observation wells). Rather than successively pumping from individual wells, we cycle

95 through sets of pumping wells. In this way, the regional aquifer is repeatedly stressed to

96 the fullest possible extent using existing wells.

97

98 We investigate the HT approach for estimating aquifer properties in a regional-scale ground 99 water model; the method results in both more detailed (higher resolution) and more 100 trustworthy (lower uncertainty) estimates. An improved estimate of aquifer properties is 101 necessary to improve the reliability of predictions made with a calibrated model.

102 Estimating aquifer parameters using the sequential successive linear estimator (SSLE, Zhu 103 and Yeh, 2005) with tomographic test data leads to better predictions of flow velocities and 104 estimates of total storage for the basin, compared to traditional methods. The numerical 105 analysis in this study was completed on a personal computer, demonstrating that HT 106 inversion can be implemented using existing computer resources.

108 In this work we use a synthetic regional confined aquifer to minimize unknown sources of 109 error (e.g., measurement and model errors) that would complicate the analyses. Initially, 110 we demonstrate that HT can be used on a regional scale, then we investigate the robustness

111 of the method by changing the variance in the true field, adding random error to the head 112 observations, and reducing the number of pumping events. Finally, HT was applied using 113 observations of drawdown, rather than head, to investigate the effects of unknown initial 114 and boundary conditions. 


\section{Methods}

117 We solve the HT inverse problem using the SSLE algorithm which is similar to that

118 developed by Yeh and Liu [2000] and Zhu and Yeh [2005]. The SSLE algorithm is an

119 extension of the SLE (successive linear estimator), that was developed for solving spatially

120 variable parameter inverse problems using a geostatistical framework [Yeh et al, 1996;

121 Zhang and Yeh, 1997; Hughson and Yeh, 2000]. The implementation of the SSLE used here

122 is coupled with the finite element flow model VSAFT2 [Yeh et al., 1993] (available for free

123 download at http://www.hwr.arizona.edu/yeh). We qualitatively discuss the key features of

124 this approach; details on the SSLE are found in Zhu and Yeh [2005].

125

126 Because high-resolution parameter estimates are the desired result of tomographic

127 inversion, we independently estimate parameter values ( $T$ and $S$ ) in each model element;

128 this leads to a large number of free parameters. The tomographic approach results in two

129 hurdles to overcome: 1) the large computational effort required to estimate the sensitivity

130 of model parameters model predictions at observation locations, and 2) the need for

131 additional constraints to reduce the degrees of freedom in the solution, since there are more

132 estimable parameters than calibration data (an ill-posed inverse problem).

134 The SSLE approach addresses both of these problems. First, the parameter-observation

135 sensitivities required for the inverse problem are computed using the adjoint approach

136 [Sykes et al., 1985; Sun and Yeh, 1992], rather than using the perturbation approach (as in

137 PEST or UCODE). The perturbation approach changes each parameter independently, 
138 running the model forward to compute the corresponding model prediction change. With

139 the perturbation inverse approach, a problem with 500 estimable parameters would require

140501 (forward difference) or 1001 (central difference) independent forward model runs per

141 iteration. For the adjoint approach, the effort to compute the model sensitivities is

142 proportional to the number of observation data. This benefits problems with a large number

143 of parameters and sparse observations, allowing sensitivities to be computed more

144 efficiently. Secondly, due to the geostatistical foundation upon which SSLE is built, the

145 parameters being estimated ( $T$ and $S$ ) are not allowed to vary arbitrarily in space, but rather

146 their distribution follows a geostatistical framework. Regularization (the observation that

147 parameters vary "smoothly" in space [Tikonov and Arsenin, 1977]) is also a means of

148 constraining the spatial distribution of parameters (implemented in PEST). The difference

149 between the geostatistical and regularization approaches is analogous to the distinction

150 between kriging and inverse distance as interpolation schemes. Both kriging and SSLE

151 incorporate additional geostatistical knowledge into their estimates, while Tikhonov-style

152 regularization and inverse distance squared are purely empirical approaches. The

153 geostatistical framework does have additional requirements (estimates of the mean,

154 variance and directionality of $T$ and $S$ ), but the accuracy of these a priori estimates is not

155 essential to the success of the algorithm in HT analyses [Yeh and Liu, 2000].

156

157 Description of synthetic problem

158 The synthetic confined aquifer used here was designed to be realistically complex, while

159 simple enough to allow straightforward interpretation of the results and the timely

160 execution of many runs required for the robustness analysis. The $2 \mathrm{D}$ model represents a 
161 depth-averaged heterogeneous $54 \mathrm{~km} \times 27 \mathrm{~km}$ aquifer, bounded by a river flowing west to

162 east on the western, northern and eastern boundaries and a mountain block on the southern

163 boundary (see Figure 1). The aquifer has two large bedrock outcrops which are represented

164 in the model by "islands" of no-flow cells (in Figure 1 inactive cells are gray). The finite

165 element mesh consists of 519 active square elements, each $1200 \mathrm{~m}$ on a side. The river is

166 a specified head boundary condition, ranging linearly from $1015 \mathrm{~m}$ to $1000 \mathrm{~m}$ from west to

167 east (dashed boundaries in Figure 1). Specified flux boundary conditions (inflow) were

168 used in four separate sections along the southern boundary to simulate fluxes into the

169 model domain from neighboring basins (dotted boundaries in Figure 1).

171 A random true $T$ field (Figure 2) was generated with an arithmetic mean of $300 \mathrm{~m}^{2} /$ day and

172 variance of $\ln (T)$ of 2.0, while the $S$ field had an arithmetic mean of 0.001 and variance of

$173 \ln (S)$ of 2.0. Transmissivity was assumed to be isotropic at the scale of the model elements

$174\left(T_{x}=T_{y}\right)$, but both the $T$ and $S$ fields were assumed statistically anisotropic at the scale of

175 the domain. The correlation scale was $20 \mathrm{~km}$ in the east-west direction and $8 \mathrm{~km}$ in the

176 north-south direction. The random $T$ and $S$ fields are uncorrelated; they utilized different

177 random seeds during their generation. Initial conditions were the results of a steady-state

178 simulation with no pumping.

179

180 HT was used to estimate the $T$ and $S$ fields by stressing the aquifer simultaneously with

181 multiple pumping wells in a manner analogous to municipal pumping or a "pump and treat"

182 remediation system. The synthetic well field was comprised of 70 wells: 20 pumping and

18350 observation wells. All wells were located randomly within the domain using a Latin 
184 hypercube approach to limit spatial clustering. While no specific effort was made to

185 optimize the wellfield for aquifer parameter estimation, the subset of 20 pumping wells was

186 visually selected to provide good spatial distribution of pumping wells throughout the

187 synthetic aquifer. Pumping wells were assigned to one of four events such that each

188 pumping event stressed most of the aquifer, resulting in overlap between the stressed areas

189 of different pumping events. The pumping well locations are shown as open symbols in

190 Figure 1: triangles are wells pumped in pumping event one, squares in event two, stars in

191 event three, and circles in event four. Each pumping event is an aquifer test that lasted 14

192 days, during which each of the five wells was pumped at $2000 \mathrm{~m}^{3} /$ day (367 gpm). The

193 initial hydraulic head distribution for each pumping event was the steady-state head

194 distribution. The aquifer response to each pumping event was observed at 50 observation

195 wells (filled dots in Figure 1). For this example we did not include the pumping wells from

196 the other pumping events in the set of observation wells, although in reality one would

197 include as many observation wells as possible.

198

199 Hydraulic head was sampled continuously at each observation well, but only four 200 observation times from each pumping event were used in the inversion: three at early time 201 and one at late time. These observation times were chosen to minimize the computational 202 effort in the SSLE inversion, while providing sufficient information to constrain the aquifer 203 parameter estimates. For noise-free data, observations through time at one location are 204 highly correlated and each new temporal observation contributes little new information 205 [Zhu and Yeh, 2005]. 
207 The true and estimated parameter fields were compared using spatial distribution maps, 208 scatter plots, and summary statistics. Good parameter estimates produce distributions that 209 are visually "similar" and scatter plots with data clustered along the 1:1 diagonal. High 210 correlation coefficient $(\rho)$ indicates a significant linear relationship between the values of

211 the two datasets, while high rank correlation coefficient $\left(\rho_{\text {rank }}\right)$ indicates patterns of highs

212 and lows are well correlated, regardless of numerical values [Isaaks and Srivastava, 1989].

213 The L1 and L2 norms indicate the differences in the log mean (bias), and log standard 214 deviation of the two datasets respectively [Yeh and Liu, 2000] (low norm values indicate 215 better fit).

216

217 Quantitative comparisons were also made between the true and predicted overall storage 218 for the entire basin. When managing ground water basins resources, accurate information 219 regarding the amount of water available from storage is essential. Lastly, we compared 220 observed and simulated velocity fields ( $v_{x}$ and $v_{y}$ ), which are required in transport 221 simulations. While head is diffuse by nature and therefore easy to match, leading to non222 unique solutions, solute transport is governed by advection (flow velocity, the gradient of 223 head), which is much more sensitive to aquifer property distributions. Two head 224 distributions can match observed point head measurements equally well, but their 225 corresponding flux distributions (and solute transport behaviors) may be very different. 226 Velocity field comparisons provide a measure of how useful the simulation would be for 227 making transport predictions. 
229

230

23

23 233 234 235 236 237 238

\section{Results}

\section{Estimation of $T$ and $S$ using Theis solution}

Aquifer parameters ( $T$ and $S$ ) are often estimated for real-world applications using the Theis solution for drawdown from a pumping well, even if some of its fundamental assumptions are known to be violated. The Theis solution is 2D (depth-averaged) and assumes an infinite homogeneous aquifer. We modeled the drawdown observed during each pumping event using the Theis solution to both illustrate the inappropriateness of a homogeneous solution for interpreting heterogeneous regional scale pumping tests and to provide a comparison to the HT results.

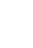

We estimated $T$ and $S$ values from the "observed" model drawdown at observation wells. For simplicity we assigned the estimated values to the location of the observation well, resulting in 50 estimates of $T$ and $S$ for each of the four pumping events. Drawdown at each observation well is due to pumping at five pumping wells. $T$ and $S$ are estimated by matching the observed drawdown to the drawdown predicted by summing the Theis solutions for the five pumping wells in a homogeneous infinite aquifer. PEST was used to minimize the sum of squared residuals between the observed and the Theis-simulated drawdown. The estimated parameter values at all 50 observation locations, for each pumping event, were then kriged to the flow simulation grid to generate the eight estimated parameter fields shown in Figures $3 a$ and $3 b$. The eight model variograms used for kriging were derived by least-squares fitting an anisotropic exponential model to the experimental variograms created from the Theis results. 
252 Due to the large domain, the simultaneous pumping of the five wells during each pumping

253 period does not cause significant interference between the wells. The radius of influence of

254 the pumping wells after 14 days of pumping (distance from the well to $1 \mathrm{~cm}$ of drawdown)

255 varies between $4 \mathrm{~km}$ and $15 \mathrm{~km}$. However, more importantly for HT, at least $1 \mathrm{~cm}$ of

256 drawdown was observed in $46 / 50$ of the observation wells during at least two of the

257 pumping events and drawdown was observed in 26/50 of the observation wells for all of

258 the pumping events.

260 We assigned parameters to observation locations, rather than pumping locations because

261 the latter would have resulted in 50 parameter estimates associated with 20 locations, 262 requiring cokriging or additional averaging to be utilized in the flow model. $T$ and $S$ 263 estimates could also have been attributed to a "representative" volume or location in the 264 aquifer, but for heterogeneous aquifers Theis-predicted values may change with time, 265 orientation, and location [Wu et al., 2005]. This makes interpretation of a representative 266 location or volume difficult, especially with the presence of boundaries. Through the 267 kriging of the intermediate point results onto the final flow simulation grid, we effectively 268 volume-averaged the Theis results in an objective and straightforward manner.

270 Visually comparing the Theis-estimated and true parameter fields (Figures 3 and 2) one can 271 see that each pair of estimated $T$ and $S$ fields is different and a poor estimate of the true 272 fields. As expected, the results of the Theis analysis are sensitive to boundaries. While Li 273 et al [2007] indicate Theis-based analyses can lead to estimates that agree, on average, with 274 tomographic results, they did not have significant boundary conditions in their problem. 
275 The curve matching produced high values of $T$ near the boundaries of the domain (see 276 Figure 3a) while high values of $S$ were consistently predicted in the south-west corner of

277 the domain (see Figure 3b). The Theis analysis has produced four different distributions of

$278 T$ and $S$ which represent the head observations associated with the four pumping events. It

279 is clear that the Theis solution doesn't give any useful information regarding the

280 distribution of parameters [Li et al. 2007], because it is a homogeneous model. The

281 hydrogeologist is left to average or decide which estimated parameter field they feel best

282 represents the true field. More realistically, Theis analyses would be performed

283 individually on each pumping test, potentially using distance drawdown to incorporate

284 multiple observation wells at one time, but the hydrogeologist may be unaware that

285 different overlapping tests can lead to markedly different results using a homogeneous 286 model such as the Theis solution.

288 Although there are obvious limitations to using the Theis solution to analyze drawdown in 289 a finite, heterogeneous domain, the exercise was done to illustrate two points. First, the 290 results from the four pumping events, which used different pumping wells (but had many 291 observation wells in common), do not produce identical or even similar results. This 292 illustrates the fact that the Theis solution doesn't simply "average out" the heterogeneity 293 around the pumping well [Wu et al, 2005]. Aside from averaging or possibly cokriging,

294 there is no straightforward way to combine the data collected in the four pumping events 295 into a single estimate (kriging does not allow for multiple values at the same location).

296 Secondly, although the shortcomings of the Theis solution are "obvious" in this synthetic 297 example, it is common practice to use Theis type curve analysis, with far less data, to 
320 Since ground water velocity controls the advective transport of solutes, velocity fields were 
321 compared as a means to quantify the quality of the SSLE calibration. The $x$ and $y$

322 components of the velocity are well correlated with small L1 and L2 norms (Figure 6). The

323 SSLE-estimated $T$ and $S$ fields would produce a reasonable estimate of advective solute

324 transport, since accurate flow velocities are the most important part of a solute transport 325 model.

327 Comparing the estimated and true total confined storage for the entire basin is another form 328 of model validation. This quantity is found by summing the product of $S$ and the area for 329 each element, for the all elements in the domain. The area of all 519 model elements is $3307.4736 \times 10^{8} \mathrm{~m}^{2}$, while the sum of the $S(\Sigma S)$ in all elements is 0.4858 for the true field, 331 giving a true total storage of $3.63 \times 10^{8} \mathrm{~m}^{3}$ (8225 acre- $\left.\mathrm{ft}\right)$. The results of SSLE inversion 332 gave $\Sigma S=0.6425$ (overestimation by $32 \%$ ), while the Theis approach gave $\Sigma S=3.029$, $3332.302,2.436$, and 3.646 for events 1 through 4, respectively; the average Theis result is 3342.853 (overestimation by 587\%). While SSLE does overestimate $S$, it is an order of 335 magnitude better than the Theis solution. We interpret that because of the lack of boundary 336 conditions in the Theis solution, it must overcompensate by overestimating the amount of

337 water coming from aquifer storage. Overestimating available storage could easily lead to 338 fallacious management decisions, by basing long-term strategies on misinformation 339 regarding available ground water.

\section{Robustness analysis}

342 We tested the robustness of the HT inversion by changing several aspects of the synthetic

343 example. First, we repeated the analysis with fewer pumping events, illustrating how HT 
344 leads to an improved estimate with additional information. Second, we added zero-mean

345 Gaussian noise with a standard deviation of $0.1 \mathrm{~m}$ to the head data, to better replicate field-

346 measured observations. Third, the HT analysis was repeated with true $T$ and $S$ fields with

347 variances of half $\left(\sigma_{\ln (T)}^{2}=\sigma_{\ln (S)}^{2}=1\right)$ and 1.5 times $\left(\sigma_{\ln (T)}^{2}=\sigma_{\ln (S)}^{2}=3\right)$ the levels of the

348 original analysis. Finally, we reformulated the HT problem in terms of drawdown to

349 minimize the effects of potentially unknown initial and boundary conditions.

351 Decreasing number of pumping events

352 One of the main strengths of HT is the ability to use multiple datasets to estimate a single

353 coherent parameter set. To illustrate the improvements from inverting multiple tests

354 together, the analysis was repeated, each time removing more pumping events from the 355 analysis. Inversion was performed using pumping events one through three, one and two, 356 and pumping event one on its own. The scatter plots of true versus estimated $T$ and $S$ for 357 each analysis are presented in Figure 7, while the results from using all four pumping 358 events are shown in Figure 4.

360 The $T$ estimate improved as more pumping events (each with different pumping wells but

361 the same observation locations) are inverted together. The cloud of points, representing $T$ in

362 each element of the flow model, moves closer to the 1:1 line, as two and three pumping 363 events are jointly inverted. This type of improvement is typical when inverting 364 tomographic aquifer tests. Each pumping event adds new information to the overall 365 estimate of the aquifer parameters, but no single pumping event by itself results in better 366 parameter estimates than analyzing two datasets simultaneously. The addition of each 
367 pumping event to the inversion process produces a smaller incremental improvement to the

368 estimated parameters than the last addition, illustrating the diminishing returns off

369 including similar data. Addition of a fourth pumping event noticeably decreases the quality

370 of the estimated $T$ field, while the quality of the estimated $\mathrm{S}$ field remains approximately

371 the same, as can be seen in the summary statistics in Table 1. Using all four pumping

372 events together may not produce optimal results for both parameters (in a non-synthetic

373 case this would be difficult to quantify), but the SSLE results remain a very good estimate

374 of the parameter distributions. In all scenarios, we used an SSLE convergence criterion of

375 a $5 \%$ relative change in the estimated parameter variance.

376

377 Random error added to observations

378 For the baseline analysis, the observations were noise-free. In this case we corrupted the 379 data with unbiased Gaussian noise with a standard deviation of $0.1 \mathrm{~m}$, to simulate more 380 realistic observations. Corrupting the observations smooths the parameter estimates, 381 however, the estimated parameter fields still generally agree with the true fields (Figure $3828 \mathrm{a}$ ), as can be seen by the high $\rho_{\text {rank }}$ values. Corrupting the data effectively decreases the 383 pumping well radius of influence (decreasing the signal to noise ratio), resulting in fewer 384 observation wells with significant drawdown signal, and increasing the scatter of the 385 predicted flow velocities (Figure $8 \mathrm{~b}$ ). For the noisy data analysis the same four observation 386 times were used from each observation well and the same convergence criterion was used.

387 This criterion aims at avoiding perfect fits between the observed and simulated heads at the 388 observation wells; this is useful when the observations are noisy. 
390 The data can be smoothed before using them in the inversion process (e.g., with a moving

391 average or wavelet smoothing) or the forward and inverse models will effectively do the

392 smoothing, because the models cannot perfectly match noisy data. To improve the

393 convergence of the inverse method, unexplained (especially biased) noise should be

394 investigated and dealt with if possible, to reduce its impact on the inverse solution [Xiang

395 et al, 2008].

396

397 Different variances in true T and S fields

398 In the previous cases, the true $T$ and $S$ fields were generated for a variance in $\ln (T)$ and

$399 \ln (S)$ of 2 . Here we examine the effect of using a smaller and larger log variance $\left(\sigma^{2} \ln (T)\right.$ and $400 \sigma_{\ln (S)}^{2}$ of 1 and 3$)$. Increasing the variance in the true field resulted in much poorer 401 parameter estimates. Both $\rho$ and $\rho_{\text {rank }}$ are smaller and the norms and larger (see columns 7 402 and 8 in Tables $1 \mathrm{a}$ and $1 \mathrm{~b})$. The larger $T$ and $S$ parameter ranges associated with the larger 403 variances are more difficult to estimate. As expected, the parameter estimates from the 404 case with a lower variance are more accurately estimated (the true parameter fields are 405 smoother) due to less nonlinear relationship between the head and the parameters [Yeh et 406 al, 1996].

Drawdown-based estimation

409 For all previous analyses, the true initial conditions were used and the boundary conditions

410 used to generate the initial condition were also used in the inverse model. In a real world 411 case, aquifer tests are rarely begun from equilibrium and the aquifer's boundary conditions 412 are often poorly known, therefore a scenario was performed where these were specified 
413 incorrectly.

414

415 A zero-drawdown specified head boundary condition was specified at all elements around

416 the outside edge of the domain - even for the specified flux and no-flow boundary

417 conditions in the true model (the two bedrock outcrops were still specified as no-flow). At

418 all the observation locations, the drawdown from the pre-test condition was used in place

419 of the simulated head. The results from this exercise, shown in Figure 9a and summarized

420 in column 6 of Tables 1a and 1b, indicate that very good results are still obtainable, even

421 when the initial or boundary conditions are poorly known. The predicted velocity

422 components (Figure 9b) are not as good as in the case where the initial condition and

423 boundary conditions are perfectly known, but the prediction is still reasonable, indicated by

424 the high $\rho_{\text {rank }}$ values.

425

426 Discussion

427

428 While a synthetic study can never take into account all the uncertainty potentially present

429 in real-world field problems, such as the potential mis-characterization of a

430 hydrologic system, it can isolate the issues related to data availability and aquifer test

431 design. In this case we have used the same model type and grid to compute the "true"

432 and inverse solutions, therefore there is no estimation error due to epistemic

433 uncertainty.

434 


\section{Viability of other methods}

436 This work stresses the benefits of using tomographic aquifer tests, and their inversion can

437 be carried out with a variety of different tools. All the results computed here were done 438 using SSLE and the finite element 2D flow model VSAFT2. Less than 10 iterations in 439 SSLE were needed to meet the specified convergence criterion.

441 Qualitative comparisons of the possible combinations of different "machinery" that could

442 be used to implement the HT inversion outlined here is beyond the scope of this paper, but 443 a similar implementation could be done using public domain software such as MODFLOW 444 [Harbaugh, 2005], PEST, or UCODE which utilize the perturbation approximation to the 445 sensitivity.

446

447 If other methods are used and aquifer properties in each element of the forward model are 448 estimated, then a regularization technique must be employed to reduce the effects of over449 parameterization. One could effectively increase the number of observations by adding 450 regularization "observations" that the parameter distribution is smooth. Alternatively, one 451 could decrease the number of parameters being estimated. This can be accomplished using 452 a pilot point method [RamaRao et al., 1995], where kriging fills in the model grid with 453 aquifer parameters from a smaller set of estimated values. Another means of accomplishing 454 this is through the singular value decomposition threshold method [Doherty, 2007], where 455 only those parameters with large singular values in the estimation process are included. 456 This reduces the dimensionality of the inverse problem without choosing a priori which 457 parameters are more important, or where pilot points should be located. 
459 Kalman filters are another class of candidate inversion algorithm; they are popular in

460

461

462

463

466

467

468

470

472

473

474

475

476

477

478

479

480 control and systems engineering, and have been applied hydrologic problems in different ways [Chen and Zhang, 2006; Goegenbeur and Pauwels, 2007]. They are more general than non-linear least squares, since model and measurement noise can be incorporated directly into the inversion process, obviating the need for smoothing noisy data, but they do not have any means of incorporating the spatial correlation between the parameters into the estimation process, as SSLE does.

\section{Conclusions}

Based on the numerical experiments performed on the given synthetic regional domain, transient HT inversion using the SSLE is shown to work well for estimating the aquifer parameters $T$ and $S$ on a regional scale. While all the simulations performed in this work have been done using the SSLE adjoint-based inverse method, this is not the only option.

We address the test scale issue that arises from applying HT to a basin-scale problem by using multiple wells distributed across the basin in each pumping event. We feel this is a realistic way to address the scale problem in a manner that can potentially be applied to monitored municipal or treatment wellfields.

The tomographic approach to analyzing aquifer test data could potentially be used on existing monitoring data. In many basins there are collections of operational data and numerous aquifer tests which have been conducted through time, which may not provide a 
481 great deal of useful basin-wide information individually, but when analyzed together, they

482 can create a whole which is greater than the sum of the parts. Results of this study appear

483 to echo the call by Yeh and Lee [2007]: It is time to change the way we collect and analyze

484 data for aquifer characterization.

485

486 Acknowledgments

487 This is an extension of a term project for the advanced subsurface hydrology class 488 (HWR535) offered at Department of Hydrology and Water Resources at the University of

489 Arizona by the last author; it was partially funded by a SERDP grant (ER-1365)

490 subcontracted through University of Iowa, and NSF IIS-0431079. We would like to thank

491 Michael Fienen and one anonymous reviewer for their detailed and insightful comments. 
492

493

494

495

496

497

500

501

502

503

504

505

506

507

508

509

510

511

512

513 Gottlieb, J., and P. Dietrich (1995), Identification of the permeability distribution in soil by

514 hydraulic tomography, Inverse Problems, 11, 353-360. 
515 Hao, Y., T.-C. J. Yeh, J. Xiang, W. A. Illman, K. Ando, K.-C. Hsu (2008), Hydraulic

516 tomography for detecting fracture connectivity, Ground Water, in press.

517 Harbaugh, A. W. (2005), The US Geological Survey modular ground-water model, the 518 ground-water flow process, in USGS Techniques and Methods 6-A16.

519 Hughson, D.L., and T.-C.J. Yeh (2000), An inverse model for three-dimensional flow in 520 variably saturated porous media, Water Resources Research, 36(4), 829-839.

521 Illman, W. A., X. Liu, and A. Craig (2007), Steady-state hydraulic tomography in a 522 laboratory aquifer with deterministic heterogeneity: Multi-method and multiscale validation of hydraulic conductivity tomograms, Journal of Hydrology, 341(3-4), $222-244$.

Isaaks, E. H., and R. M. Srivastava (1989), An Introduction to Applied Geostsatistics, 526 Oxford.

527 Konikow, L. F., and J. D. Bredehoeft (1992), Groundwater models cannot be validated, 528 Advances in Water Resources, 15(1), 75-83.

529 Li, W., A. Englert, O. A. Cirpka, J. Vanderborght, and H. Vereecken (2007), Two530 dimensional characterization of hydraulic heterogeneity by multiple pumping tests, 531 Water Resources Research, 43(4), W04433.

532 Liu, S., T.-C. J. Yeh, and R. Gardiner (2002), Effectiveness of hydraulic tomography: 533 sandbox experiments, Water Resources Research, 38(4)WR000338.

534 Liu, X., W. A. Illman, A. J. Craig, J. Zhu, and T.-C. J. Yeh (2007), Laboratory sandbox 535 validation of transient hydraulic tomography, Water Resources Research, 43(5), W05404.Oreskes, N., K. Shrader-Frechette, and K. Belitz (1994), Verification, 
validation and confirmation of numerical models in the earth sciences, Science, 243(5147), 641--646.

Poeter, E. P., M. C. Hill, E. R. Banta, S. Mehl, and S. Christensen (2005), UCODE_2005 and six other computer codes for universal sensitivity analysis, calibration, and uncertainty evaluation, in USGS Techniques and Methods 6-A11.

RamaRao, B. S., A. M. LaVenue, G. de Marsily, and M. G. Marietta (1995), Pilot point methodology for automated calibration of an ensemble of conditionally simulated transmissivity fields, 1, Theory and computational experiments, Water Resources Research, 31(3), 475-493.

Straface, S., T.-C. J. Yeh, J. Zhu, S. Troisi, and C. H. Lee (2007), Sequential aquifer tests at a well field, Montalto Uffugo Scalo, Italy, Water Resources Research, 43, W07432.

Sun, N.-Z., and W. W.-G. Yeh (1992), A stochastic inverse solution for transient groundwater flow: Parameter identification and reliability analysis, Water Resources Research, 28(12), 3269-3280.

Sykes, J. F., J. L. Wilson, and R. W. Andrews (1985), Sensitivity analysis for steady state groundwater flow using adjoint operators, Water Resources Research, 21(3), 359371.

Tikhonov, A. N., and V. Y. Arsenin (1977), Solutions of Ill-Posed Problems, Wiley.

Tosaka, H., K. Masumoto, and K. Kojima, (1993), Hydropulse tomography for identifying 3-D permeability distribution, in Proceedings of the $4^{\text {th }}$ Annual International Conference on High Level Radioactive Waste Management.

Vasco, DW, H, Keers, and K. Karasaki (2000), Estimation of reservoir properties using transient pressure data: An asymptotic approach, Water Resources Research, 36 
(12): 3447-3465.

561 Vesselinov, V. V., S. P. Neuman, and W. A. Illman (2001), Three-dimensional numerical inversion of pneumatic cross-hole tests in unsaturated fractured tuff: 2. Equivalent parameters, high-resolution stochastic imaging and scale effects. Water Resources Research, 37(12), 3019-3042.

Wu, C. -M., T.-C. J. Yeh, J. Zhu, T. H. Lee, N.-S. Hsu, C.-H. Chen, and A. Folch-Sancho (2005), Traditional analysis of aquifer tests: Comparing apples to oranges?, Water Resources Research, 41(9), W09402.

Xiang, J., T.-C. J. Yeh, C.-H. Lee, K.-C. Hsu, and J.-C. Wen (2008), A new estimator and a guide for hydraulic tomography analysis, Ground Water (in press).Yeh, T.-C.J. (1992), Stochastic modeling of groundwater flow and solute transport in aquifers, Journal of Hydrologic Processes, 6, 369-395.

Yeh, T.-C. J., R. Srivastava, A. Guzman, T. Harter (1993), A numerical-model for waterflow and chemical-transport in variably saturated porous-media. Ground Water. 31 (4): $634-644$.

Yeh, T.-C. J., M. Jin, and S. Hanna (1996), An iterative stochastic inverse method: conditional effective transmissivity and hydraulic head fields, Water Resources Research, 32(1), 85-92.

Yeh, T.-C. J., and C. H. Lee (2007), Time to change the way we collect and analyze data for aquifer characterization, Ground Water 45 (2): 116-118.

Yeh, T.-C. J., C. H. Lee, K.-C. Hsu, and Y.-C. Tan (2007), "Fusion of Active and Passive Hydrologic and Geophysical Tomographic Surveys: The Future of Subsurface 

Hyndman, F. D. Day-Lewis, and K. Singha, AGU monograph.

584 Yeh, T.-C. J., and S. Liu (2000), Hydraulic tomography: Development of a new aquifer test method, Water Resources Research, 36(8), 2095-2105.

586 Yeh, T.-C. J., and P. A. Mock (1996), Structured approach for calibrating steady-state 587 ground-water flow models, Ground Water, 34(3), 444-450.

588 Zhang, J., and T.-C. J. Yeh (1997), An iterative geostatistical inverse method for steady 589 flow in the vadose zone, Water Resources Research, 33(1), 63-71.

590 Zhu, J. and T.-C. J. Yeh (2005), Characterization of aquifer heterogeneity using transient hydraulic tomography, Water Resources Research, 41(7), W07028.

592 Zhu, J. and T.-C. J. Yeh (2006), Analysis of hydraulic tomography using temporal moments 593 of drawdown recovery data, Water Resources Research, 42(2), W02403. 
594 TABLE

595

\begin{tabular}{c|ccccccc|c|c|}
\cline { 2 - 8 } \multicolumn{1}{c|}{} & \multicolumn{7}{c|}{$\sigma^{2} \ln (T)=2.0$} & \multicolumn{1}{c|}{} \\
\cline { 2 - 8 } 1a & Event 1 & Events 1-2 & Events 1-3 & Events 1-4 & $\begin{array}{c}\text { Noisy } \\
\text { observations }\end{array}$ & $\begin{array}{c}\text { Drawdown }+ \\
\text { Incorrect BC }\end{array}$ & $\sigma^{2}{ }_{\ln (T)}=1.0$ & $\sigma^{2} \ln (T)=3.0$ \\
\hline$\rho$ & 0.53 & 0.77 & 0.87 & 0.73 & 0.69 & 0.82 & 0.86 & 0.011 \\
$\rho_{\text {rank }}$ & 0.77 & 0.86 & 0.89 & 0.85 & 0.82 & 0.84 & 0.93 & 0.033 \\
L1 & 0.85 & 0.56 & 0.46 & 0.58 & 0.72 & 0.60 & 0.27 & 1.39 \\
L2 & 1.25 & 0.58 & 0.42 & 0.64 & 0.89 & 0.87 & 0.13 & 2.90 \\
\hline
\end{tabular}

596

597

\begin{tabular}{|c|c|c|c|c|c|c|c|c|}
\hline \multirow[b]{2}{*}{$1 b$} & \multicolumn{6}{|c|}{$\sigma^{2} \ln (S)=2.0$} & \multirow[b]{2}{*}{$\sigma_{\ln (S)}^{2}=1.0$} & \multirow[b]{2}{*}{$\sigma^{2} \ln (S)=3.0$} \\
\hline & Event 1 & Events 1-2 & Events 1-3 & Events 1-4 & $\begin{array}{c}\text { Noisy } \\
\text { observations }\end{array}$ & $\begin{array}{l}\text { Drawdown + } \\
\text { Incorrect BC }\end{array}$ & & \\
\hline$\rho$ & 0.37 & 0.77 & 0.81 & 0.80 & 0.65 & 0.33 & 0.87 & 0.083 \\
\hline$\rho_{\text {rank }}$ & 0.67 & 0.73 & 0.74 & 0.77 & 0.76 & 0.71 & 0.87 & 0.031 \\
\hline $\mathrm{L} 1$ & 1.14 & 0.75 & 0.67 & 0.70 & 0.75 & 0.95 & 0.33 & 1.38 \\
\hline L2 & 2.20 & 1.13 & 1.01 & 0.96 & 1.05 & 1.46 & 0.22 & 3.07 \\
\hline
\end{tabular}

598

599 Table 1Comparison of summary $T$ (1a) and $S(1 \mathrm{~b})$ statistics for different SSLE inverse

600 solutions, $\rho$ and $\rho_{\text {rank }}$ are the correlation and rank correlation coefficients, L1 and L2 are

601 norms indicating bias and error in standard deviation respectively.

602 
603

604 FIGURES

605

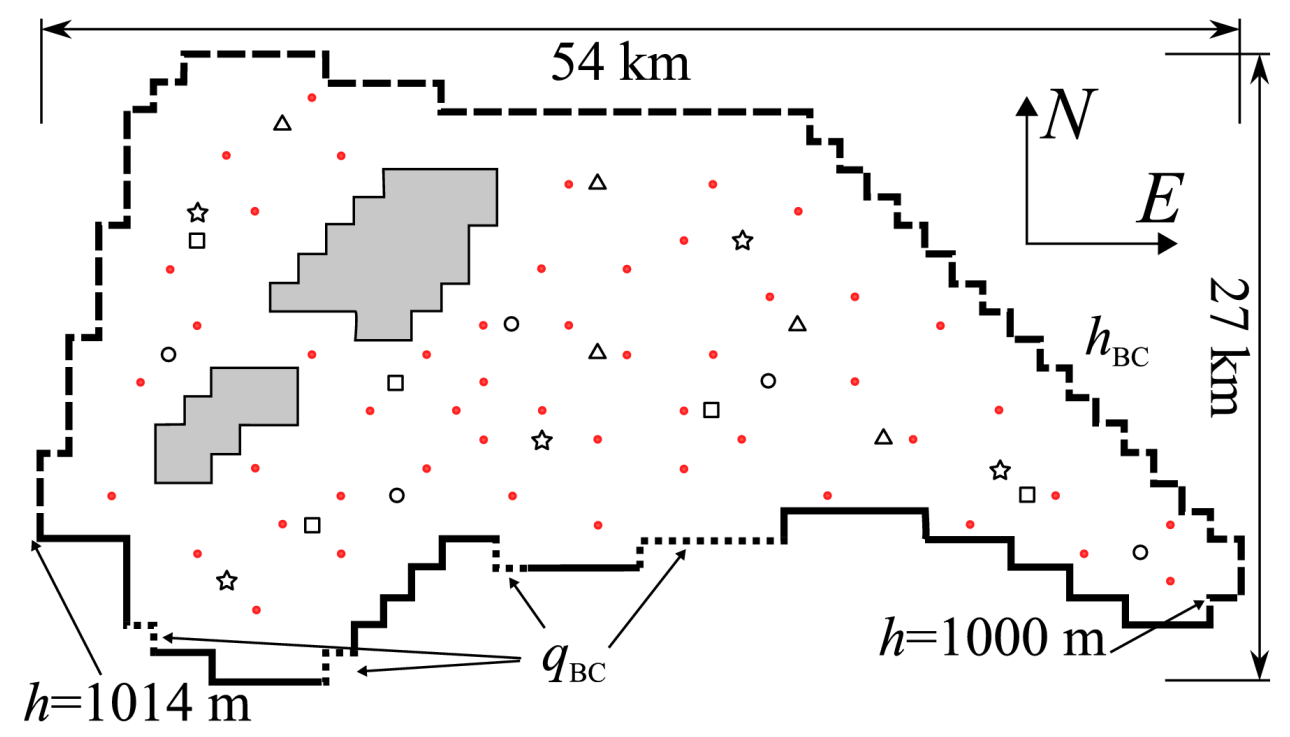

606 Fig 1 Map of domain showing pumping (open) and observation (solid) locations;

$607 \Delta=$ event $1, \square=$ event 2 , star=event $3, \circ=$ event 4 . Dashed boundary is specified head, 608 dotted boundary is specified non-zero flux, and solid boundary is no-flow.

609

610 


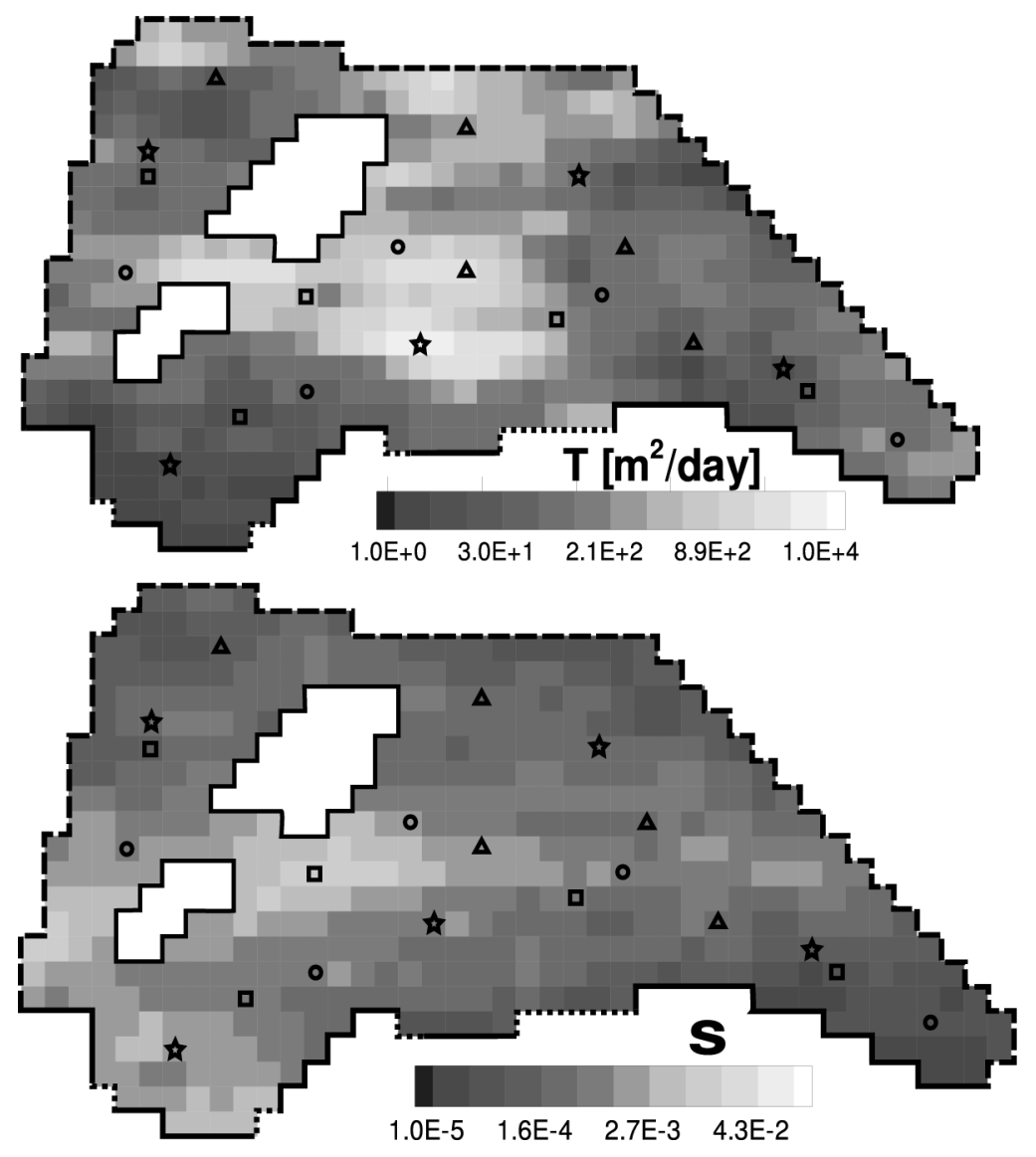

612 Fig 2: Map of true randomly-generated $T$ field with $\sigma^{2} \ln (T)=2.0$ and mean $=300 \mathrm{~m}^{2} / \mathrm{day}$,

613 and $S$ field with $\boldsymbol{\sigma}_{\ln (S)}^{2}=2$ and mean $=0.0001$.

614

615 
616

617

618

619

620 


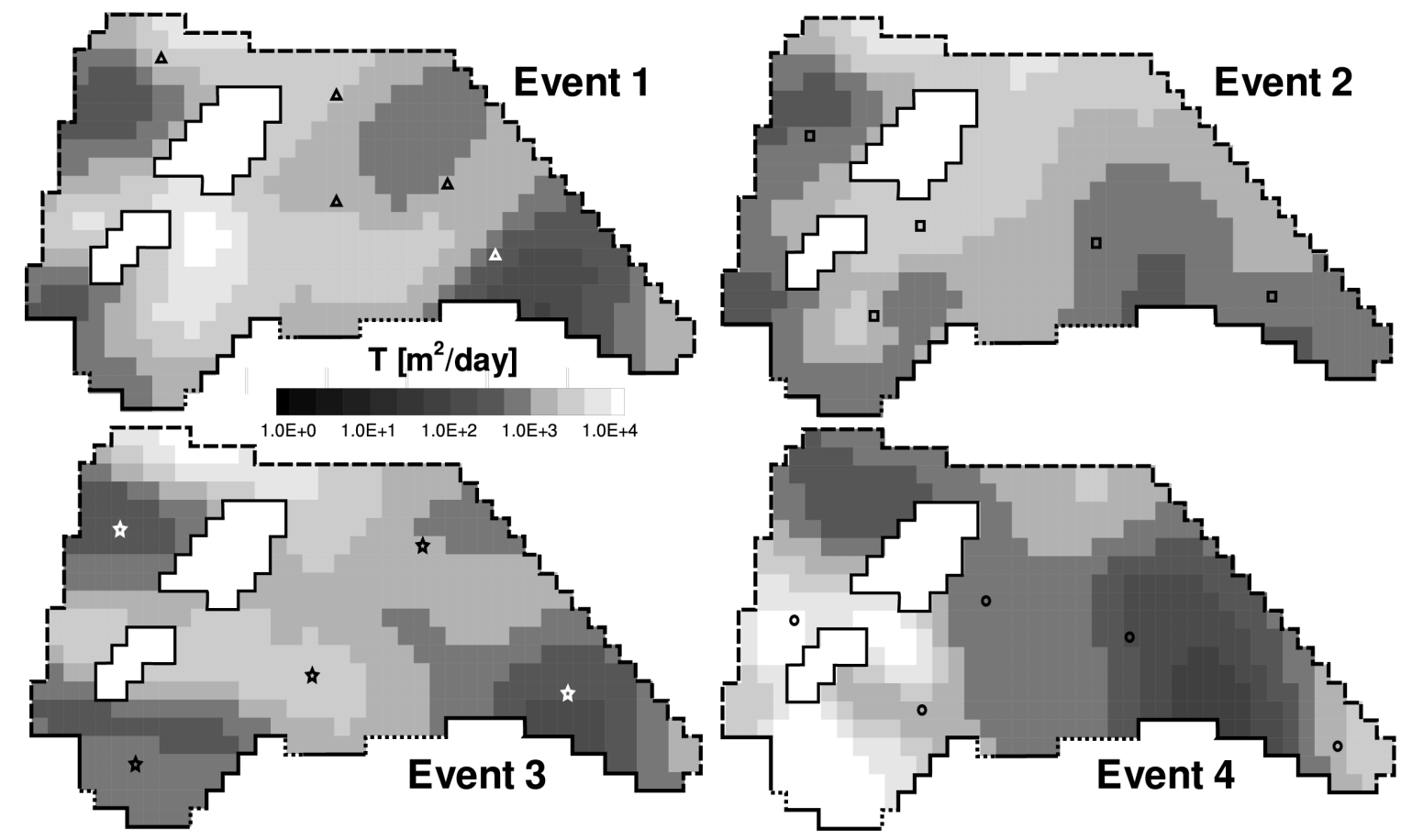

622 Fig 3a Maps of estimated $\boldsymbol{T}$ using Theis analysis

623

624 
625

626
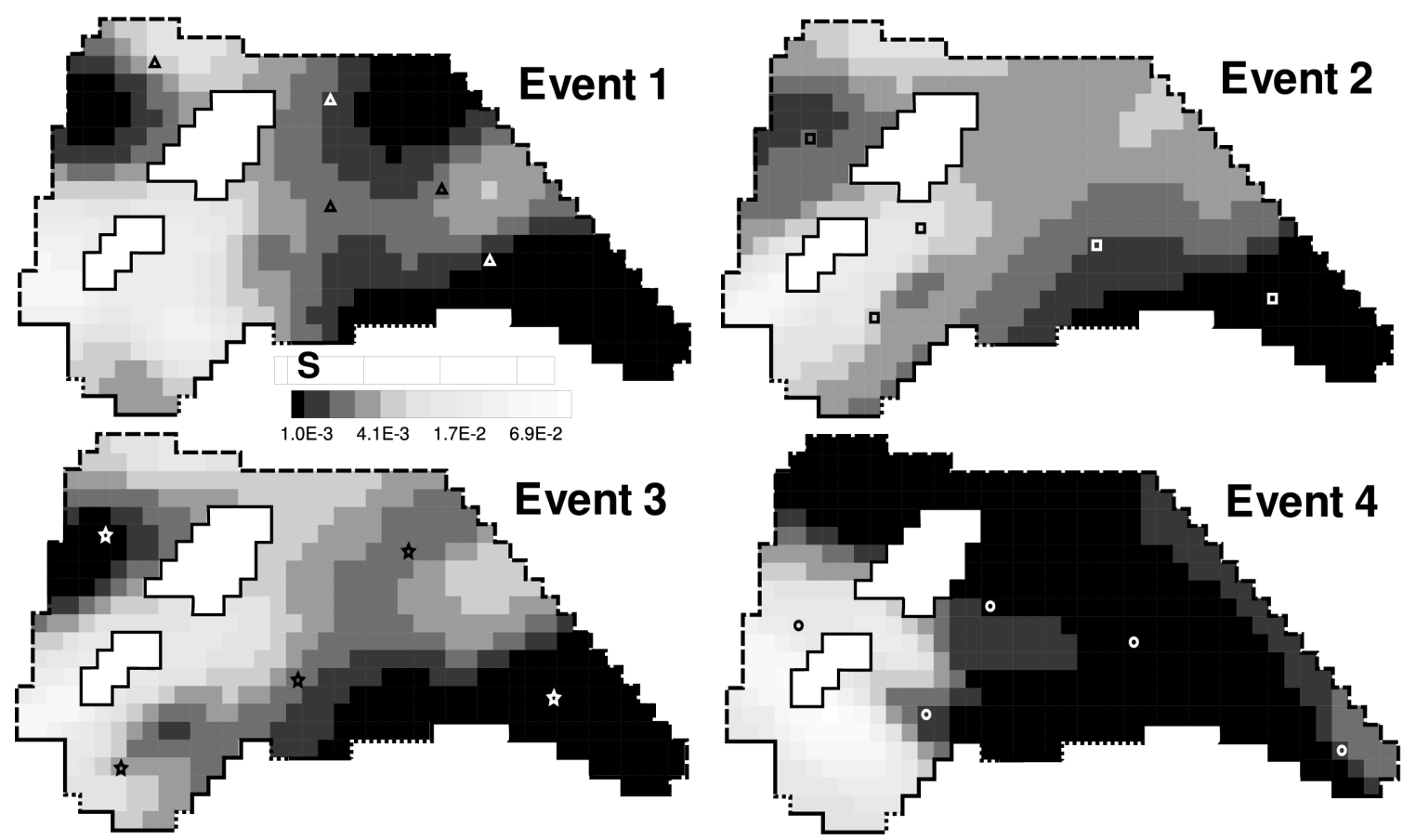

627 Fig 3b Maps of estimated $S$ using Theis analysis

628

629 
630

631

632
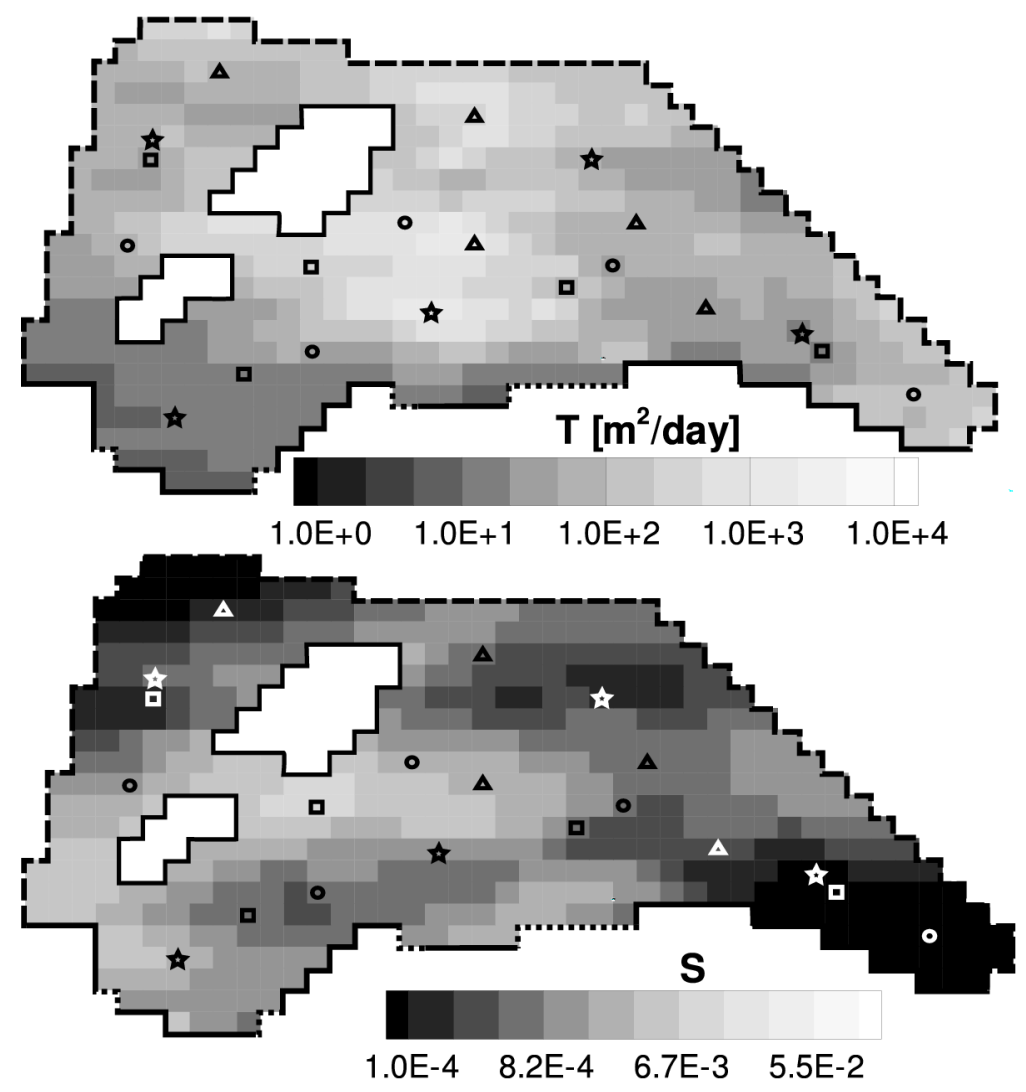

633 Fig 4 Maps of estimated $\boldsymbol{T}$ and $S$ using SSLE method (all 4 pumping events)

634

635 
636

637
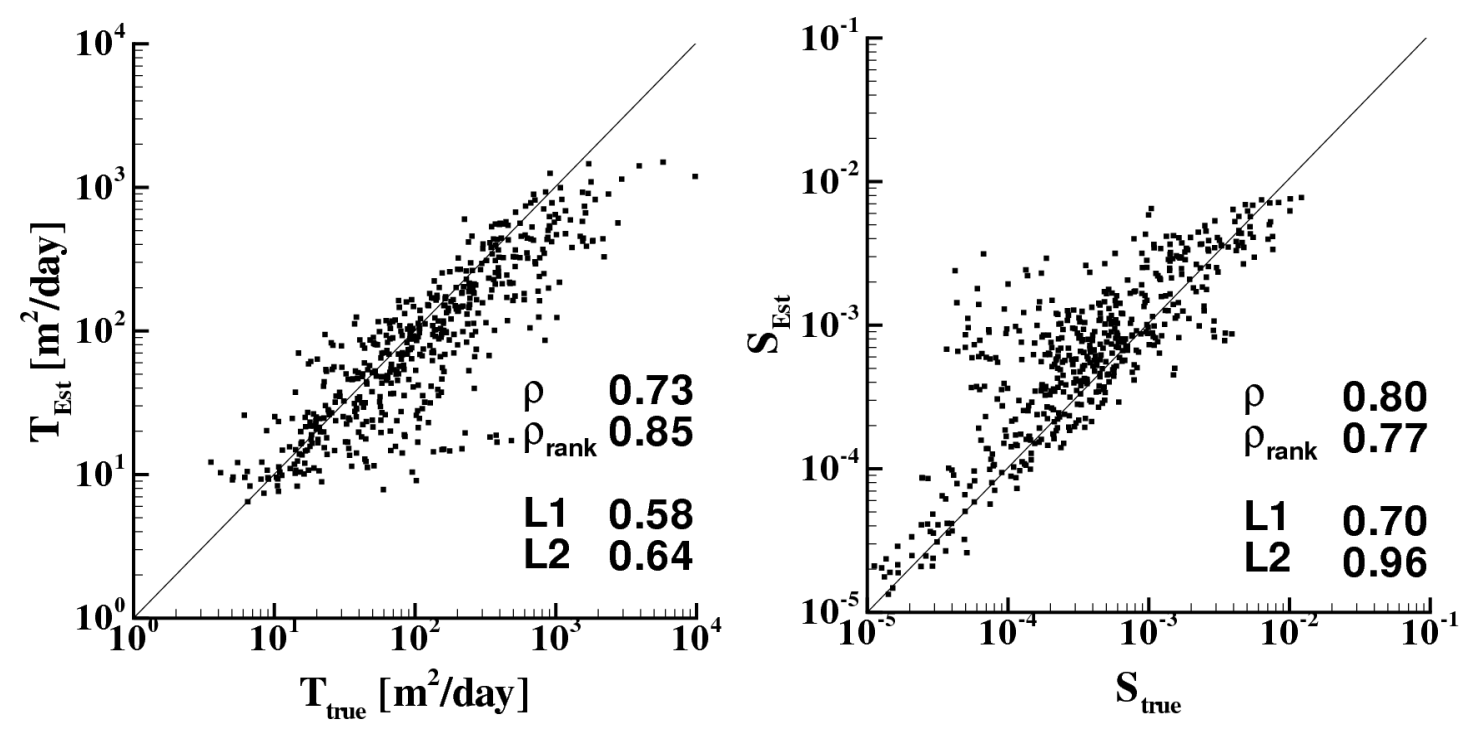

638 Fig 5 Scatterplots of estimated $\boldsymbol{T}$ and $\boldsymbol{S}$ using SSLE

639

640 
641

642
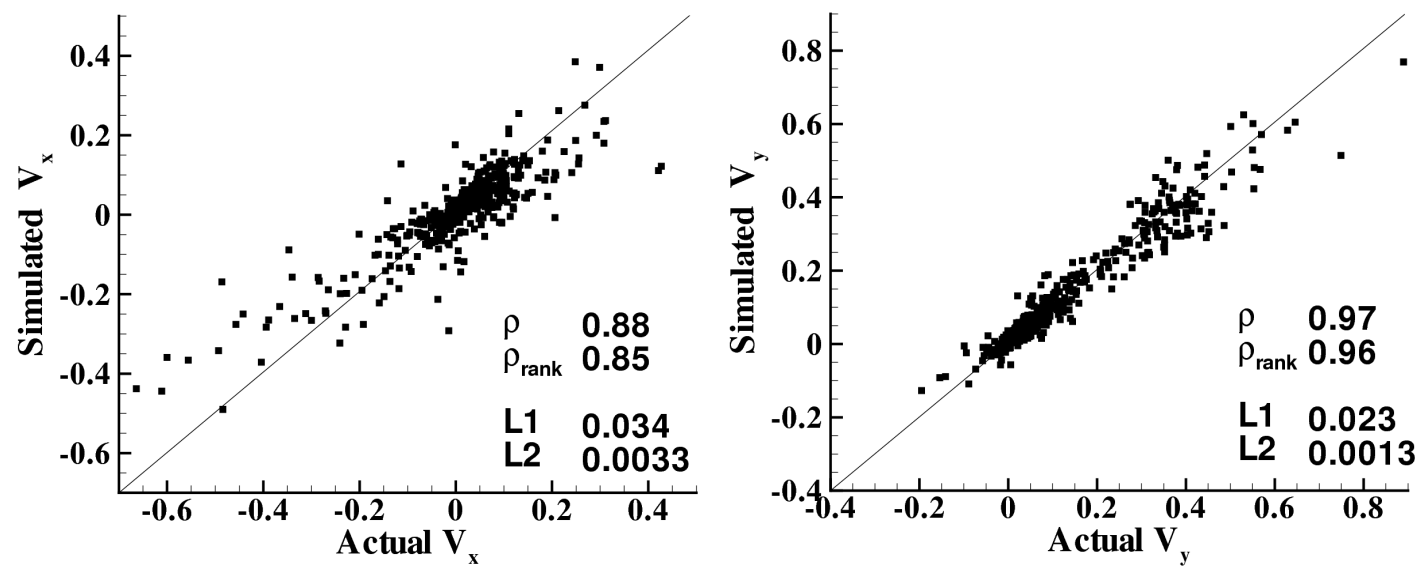

643 Fig 6 Scatterplots of $\boldsymbol{x}$ and $\boldsymbol{y}$ velocity components for SSLE-estimated $\boldsymbol{T}$ and $\boldsymbol{S}$

644

645 
646

647
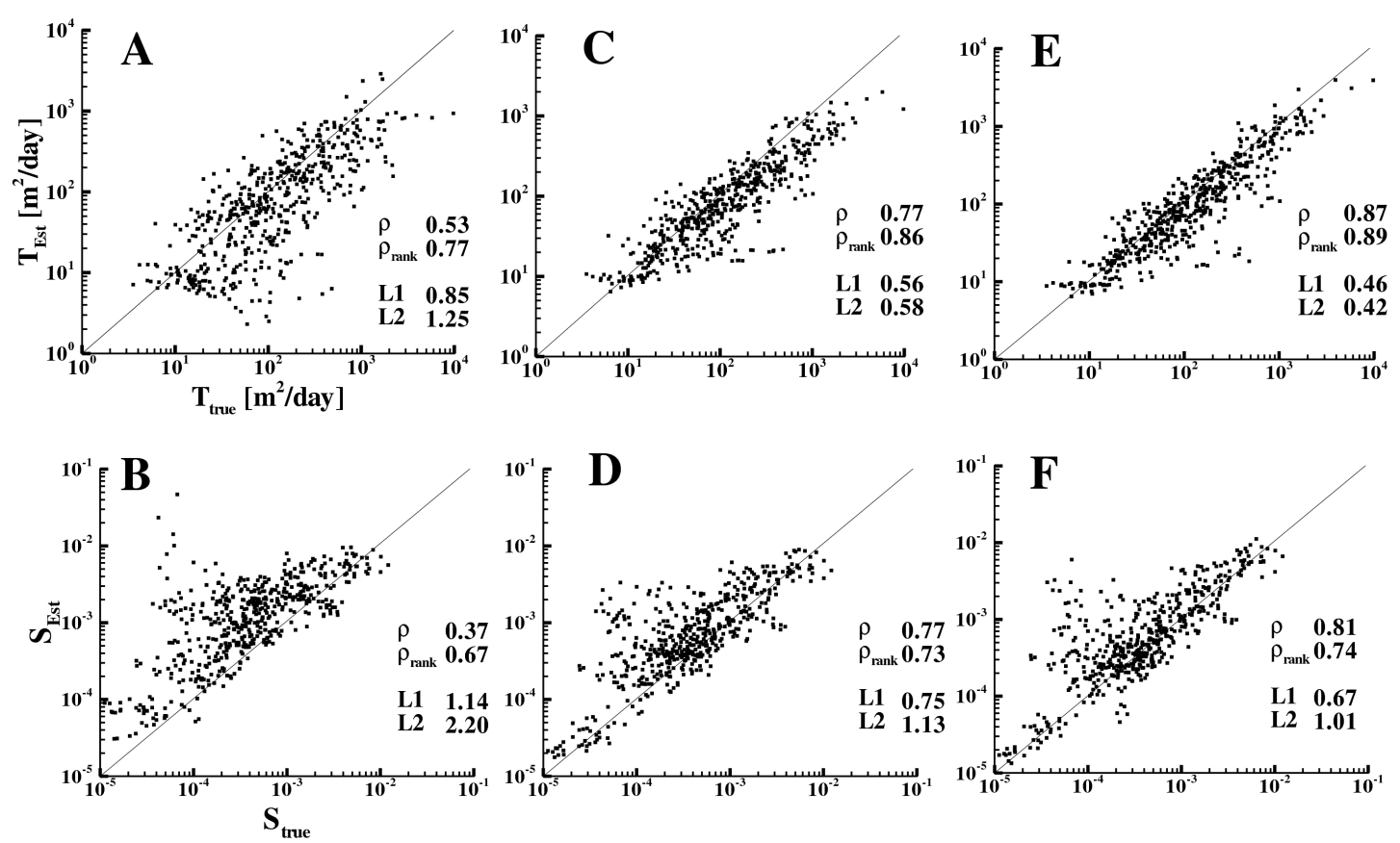

Fig 7 Scatterplots of SSLE inversion using different pumping events (top row is $T$, bottom row is $S$ ); $A, B=$ event 1 only; $C, D=$ events $1-2 ; E, F=$ events $1-3$; see Figure 5 
653

654
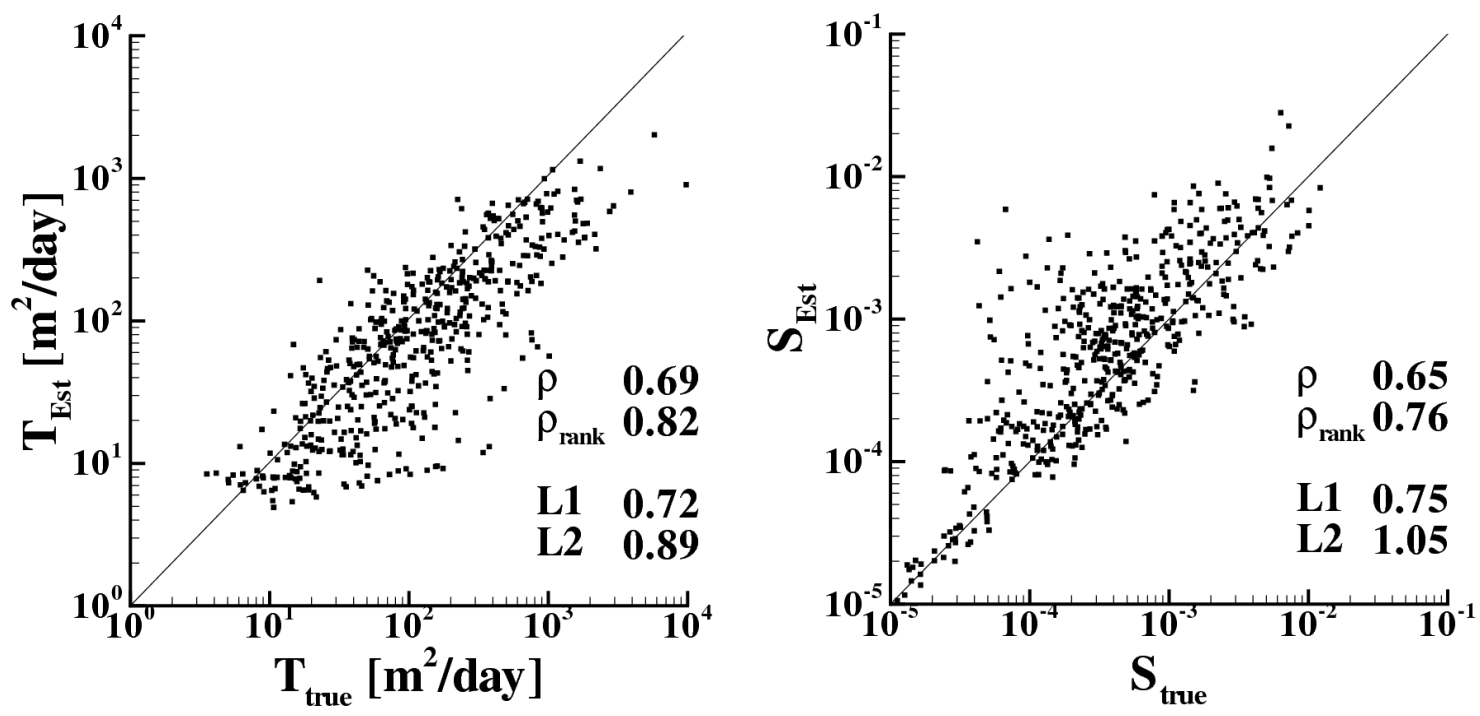

655 Fig 8a Scatterplots of SSLE-estimated $T$ and $S$ with noisy $(\sigma=0.1 \mathrm{~m})$ head observations

656

657 
658

659
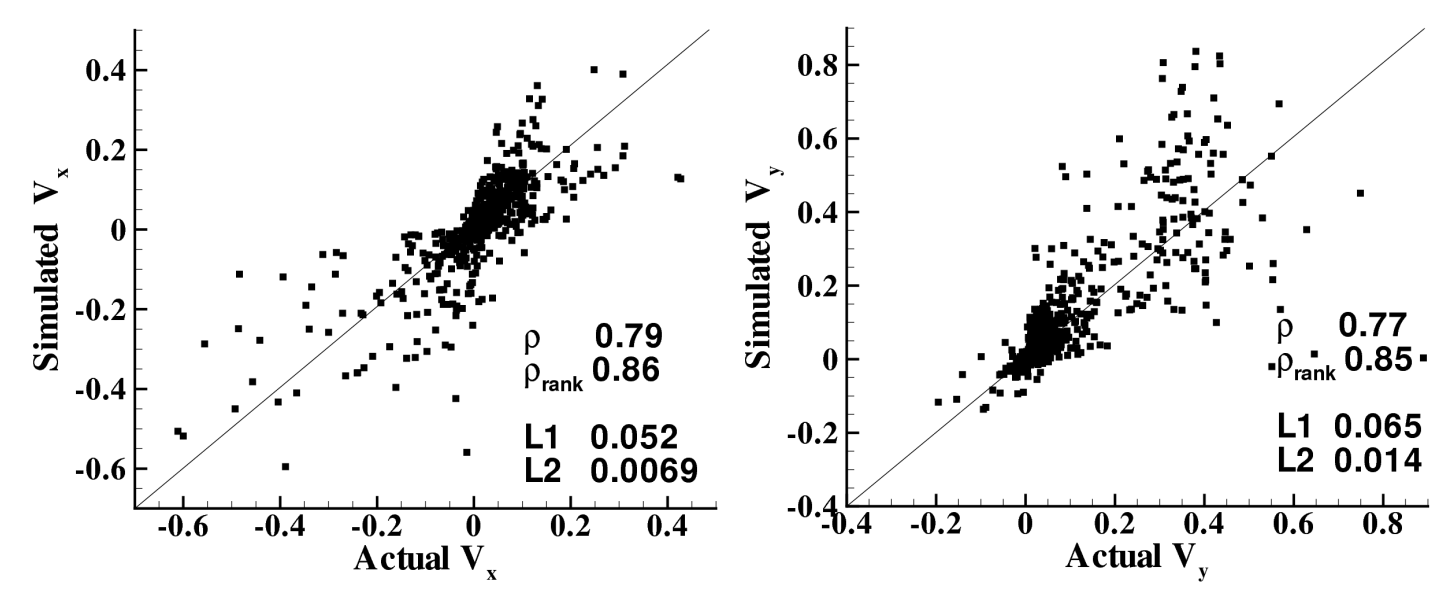

661

662 Fig $8 b$ Scatterplots of $x$ and $y$ velocity components for SSLE-estimated $T$ and $S$ using

663 noisy observations

664

665 
666

667
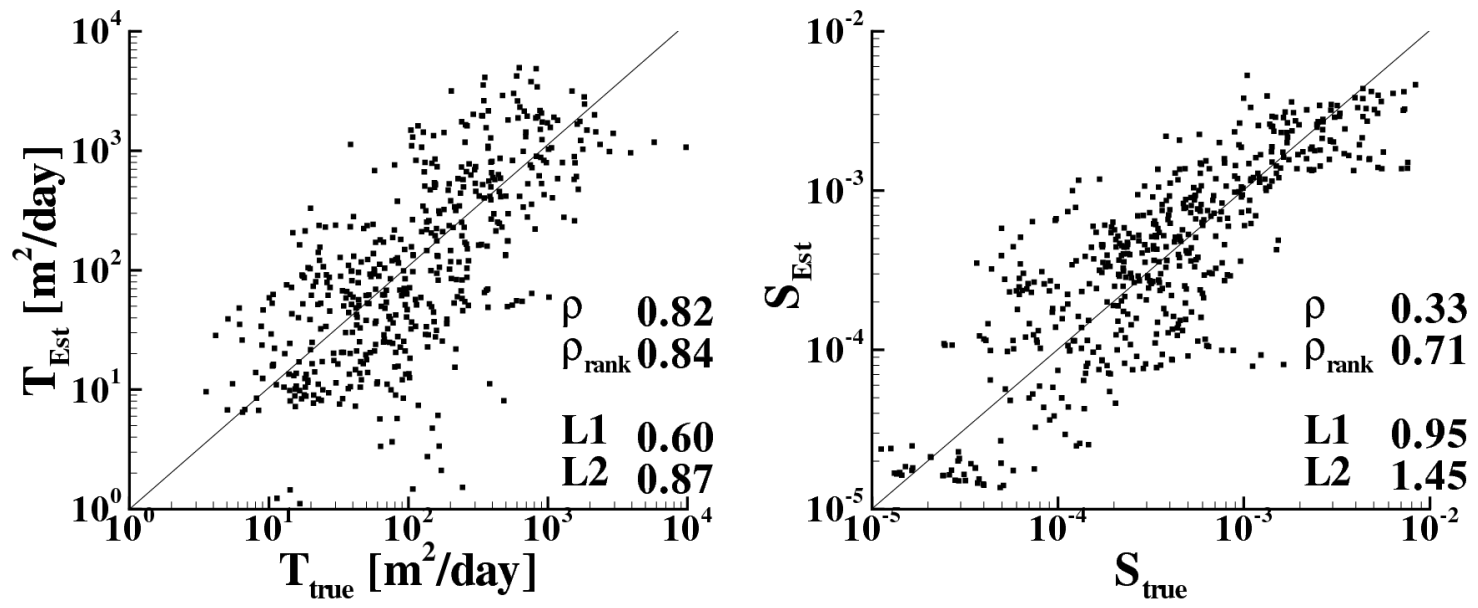

668 Fig 9a Scatterplots of SSLE-estimated $\boldsymbol{T}$ and $\boldsymbol{S}$ using drawdown and incorrect

669 boundary conditions

670

671 
61

672

673
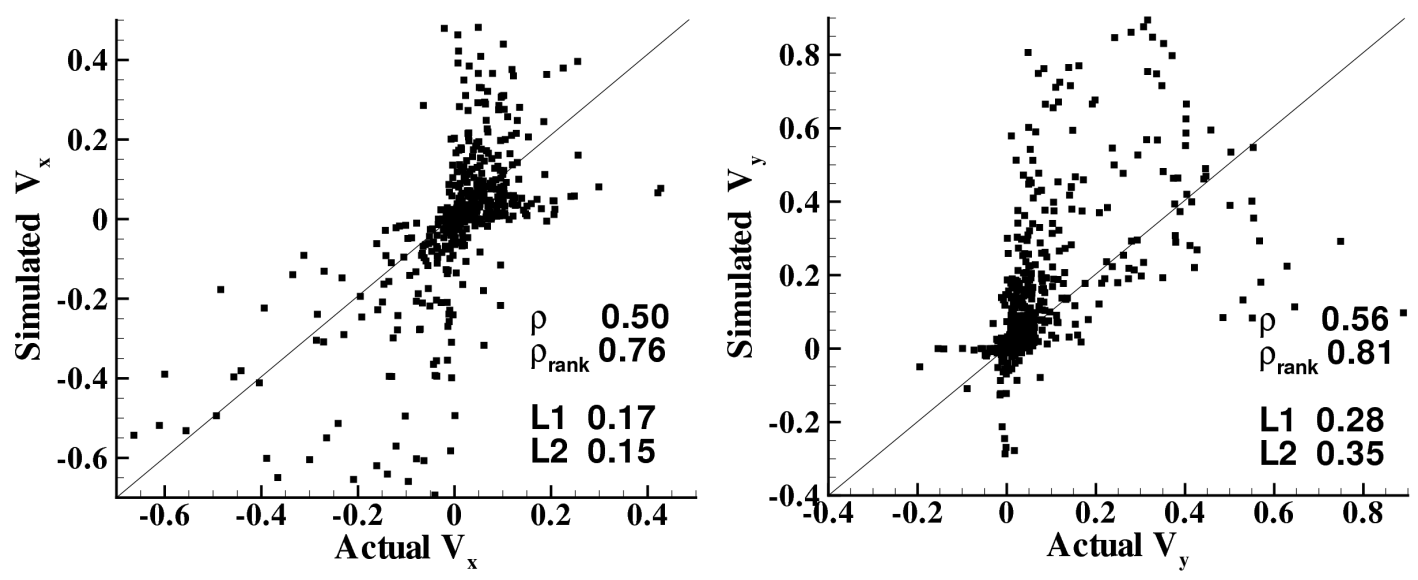

674 Fig 9b Scatterplots of $\boldsymbol{x}$ and $\boldsymbol{y}$ components of velocity for SSLE-estimated $\boldsymbol{T}$ and $\boldsymbol{S}$

675 using drawdown and incorrect boundary conditions

676

677 